out into the anterior chamber, the aqueous humour can easily get in, and disintegration, liquefaction, and absorption in due time ensue, leaving of ten a perfectly transparent capsule. But if, after a reasonable delay, opaque matter still obstructs the pupillary area, the capsule may easily be torn through by a single needle, inasmuch as its natural fragility has not been impaired by previous instrumentation. A possible objection to peripheral section of the capsule may be answered by anticipation. "Secondary" cataract is not more frequent than when the face of the capsule has been torn by the pricker or removed by forceps. Perhaps it is not so frequent.

\section{NOTES ON A CASE OF}

\section{SUPPURATING HYDATID, PROBABLY CONNECTED WITH THE LIVER; GASTROTOMY ; RECOVERY.}

BY EDWARD O. DALY, M.A., M.D. OxON., M.R.C.P., PHYSICIAN TO THE HULL ROYAL INFIRMARY.

T,C- a a the Hull Infirmary on Aug. 6th, 1885, complaining of pain across the abdomen and in the back of seven weeks' duration. His abdomen had gradually enlarged during that period, and he felt quite sure that he was his usual size round the waist two months prior to admission. Previous to his present illness he had enjoyed excellent health. On examination the abdomen was found to be tender and greatly distended, the circumference at the umbilicus being thirty-nine inches. 0 percussion a dull note was obtained over the front of the abdomen; there was no bulging at the flanks, and in this situation the note was clear and tympanitic. The dulness and tympanitic note did not change with the position of the patient. On palpation distinct fluctuation could be readily obtained. There was some cedema of the legs, the urine was normal, and the heart and lung sounds natural. The day after admission he was tapped, and a pint of brownish fluid drawn off containing a large amount of albumen and bile. This fluid, which was quite sweet when first withdrawn, had a specific gravity of 1006, and on standing turned from a brown to a greenish colour; a large amount of sediment was deposited, which was found to consist of micro-organisms in countless numbers; there were also a few crystals of leucin and tyrosin. Prior to his being tapped it was thought likely that the tumour was a hydatid cyst; the character, however, of the fluid removed seemed to negative that idea. On Aug. 27 th the tapping was repeated, twelve pints of similar fluid being withdrawn. The patient left the infirmary on Sept. 25th, and was readmitted in February, 1886, remaining an inpatient until May 31st. During this poriod he was tapped on three different occasions, seventy-eight pints of fluid being removed. The amount of bile diminished at each tapping; the albumen somewhat increased, but in other respects the fluid ramained unaltered. His health continued remarkably good; the temperature was always normal; his weight, too, remained stationary. On July $6 \mathrm{th}, 1886$, he was readmitted for the third time. Thirty-six pints of fluid were withdrawn and the abdomen carefully explored. No enlargement of the liver could be made out, and no solid growth of any kind discovered. The fluid very rapidly reaccumulated, and in a week or ten days he was as big as ever, his circumference at the umbilicus being forty-five inches. as the patient was very anxious to have an operation if there was any reasonable hope of success, a consultation was held as to the advisability of opening the abdomen. The patient's health, however, seamed so good, and the diagnosis so uncertain, that such a proceeding at that time was hardly thought justifiable. Towards the end of September he developed signs of peritonitis, with marked elevation and remission of temperature. A small quantity of fluid was removed, which for the first time was offensive. It was therefore at once decided to open the abdomen. The fluid was completely removed, and along with it shreds of membrane resembling plum-skins, which on examination were found to be pieces of hydati I membrane. The cyst cavity was then washed out with some antiseptic, and a drainage tube inserted. The patient made a rapida and complete recovery.
The following are the chief points of interest in connexion with this case:-1. The large size of the cyst, which filled the greater part of the abdomen. The cyst must either have been barren or contained only a very limited number of secondary or daughter cysts, since a comparatively small amount of membrane escaped with the fluid at the operation. No hooklets or echinococci were ever discovered in the fluid after careful examination, and it is quite probable that the cyst did not advance to that stage of development in which echinococci are formed. The examination, therefore, of the fluid withdrawn from a barren hydatid cyst in which suppuration has taken place does not necessarily assist one in arriving at a correct diagnosis. 2. Although tho liver was never definitely made out to be enlarged, either before or at the operation, the cyst was most probably connected with the liver; it at any rate at one time communicated with one of the bile ducts, as a considerable amount of bile was found in the fluid, especially in that from the first two tappings. It was, I take it, the presence of a considerable amount of bile which kept the fluid sweet after the cyst had suppurated and destroyed the pus-corpuscles, so that none were ever made out microscopically. 3. The case shows the nonadvisability of continuing to aspirate a cyst that has been tapped two or three times and quickly refills. The removal of the fluid from the cyst diminishes the pressure, and so an effusion of serum takes place within its capsule from the vessels that supply it. The consequence is that the fluid removed in the later tappings is largely composed of albumen, which the patient is ill able to dispense with. 4. How can the sudden offensiveness of the fluid be best explained? There seem two possible causes for it: either it depended on the needle used at the last tapping being septic, or on the removal of all the bile from the fluid of the cyst (no trace of which could be detected at the last tapping). The antiseptic properties of bile are well known. It may be, therefore, that as long as there was any bile in the fluid it remained sweet; as soon as it ceased to exist the fluid became offensive. Perhaps both these causes had a share in the matter.

\section{THE FUMTFIER :}

A DESCRIPTIVE ACCOUNT OF ITS CONSTRUCTION AND USE.

\section{Br ROBERT J. LEE, M.A., M.D. CANTAB,, F.R.C.P.}

Mosr substances which exist naturally in the form of fluid may be converted by heat into the form of vapour, and likewise many substances existing naturally as solids may be changed by heat into "fumes" or "smoke." For therapeutic purposes there are differences between the change of condition alluded to, as it occurs in the two cases, which are deserving of consideration. Taking opium by way of example, we perceive that we cannot convert it into vapour by making a solution of it and then boiling. The water would evaporate and leave the opium as a residue. By heating opium to a high temperature, as in the ordinary method of smoking, the substance is converted into the form of vapour by a process of slow and more or less destructive distillation. The vapour or smoke obtained from opium, thus treated, differs from opium in certain respects which have been the subject of investigation. It is enough for our purpose to recognise the fact thut the result is different in such a case from that obtained when a fluid such as water or alcohol is converted into vapour. This vapour is chemically the same as the fluid, and all that has occurred has been a change of molecular condition. Though there is little or no difficulty in converting medicinal agents from the form of fluid into that of vapour, the same facility does not exist in the case of solids. When we consider the very extensive use all over the world of the tobacco plant in the form of "vapour," or rather of fume, or "smoke," and the less extensive use, in certain countries, of opium in a similar form, it may reasonably be asked whether the subject of the conversion of medicinal agents of well-known potentiality from the solid state to that of "smoke" is not deserving of attention, as affording us another mode of administering them therapeutically. The difficulty to be overcome is a very simple one. We require to support a slow process of combustion in the substance under treatment which is practically a process of destructive distillation; and just as in the pre- 
paration of gas from coal the heat is carefully regulated, ard the changes taking place within the retorts are very different from those which occur in the ordinary burning of coal, so in the case of opium, stramonium, and other substances our object is to convert them into fumes by regulating the heat and limiting the supply of air, so as to change the molecular condition without destructive combustion. So far as the leaves, stalks, or other parts of medicinal plants are concerned, we may deal with them in the same way as with tobacco, and convert them into "fumes" by slow combustion-that is, by ignition, and by forced induction of atmospheric air. But substances like opium, calomel, arsenic, \&c., which are not readily ignited, require to be treated some-

FrG. 1.

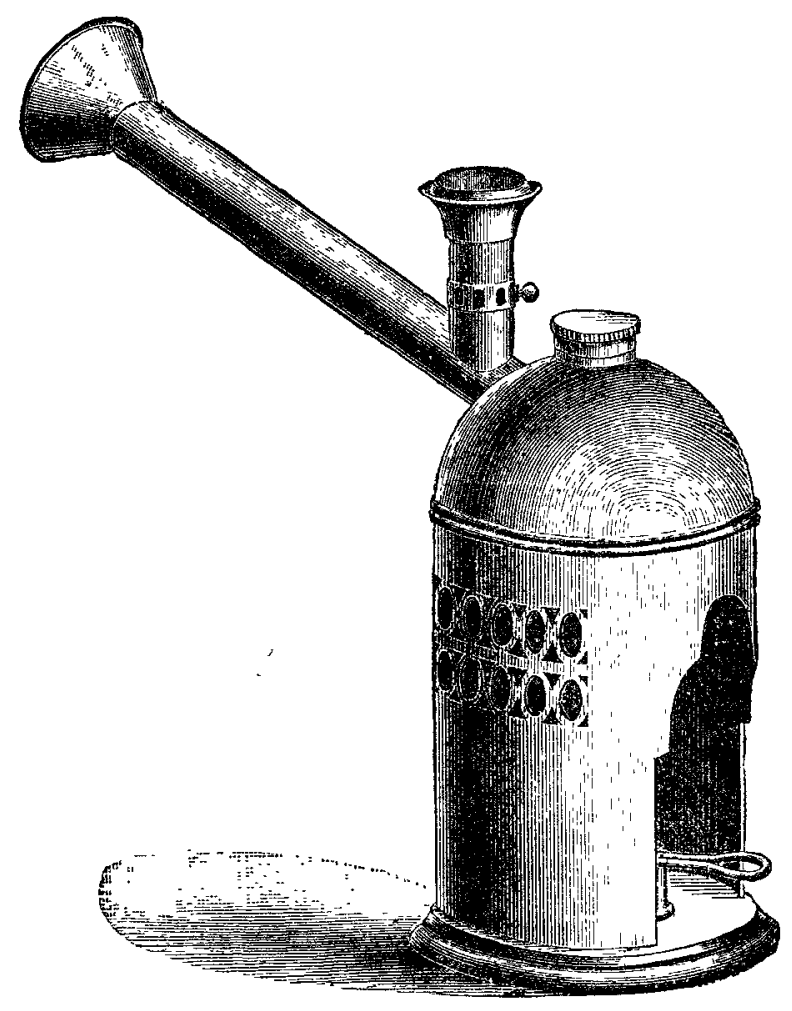

what differently. By combining them with some substance, such as pure carbon, as is done in the familiar instance of pastilles, the fumes of the substance can be obtained. It must be clear from what has been said that we can generally induce slow combustion in any substance if we have the means of promoting it by an induced current of air, and I propose to explain briefly how this may conveniently be done by the action of a small jet of steam. (Fig. 1 is a representation of the fumifier.)

By careful attention to the principle on which the steam jet acts when playing in the axis of a tube, as is shown in Fig. 2, we can induce combustion to take place in the way

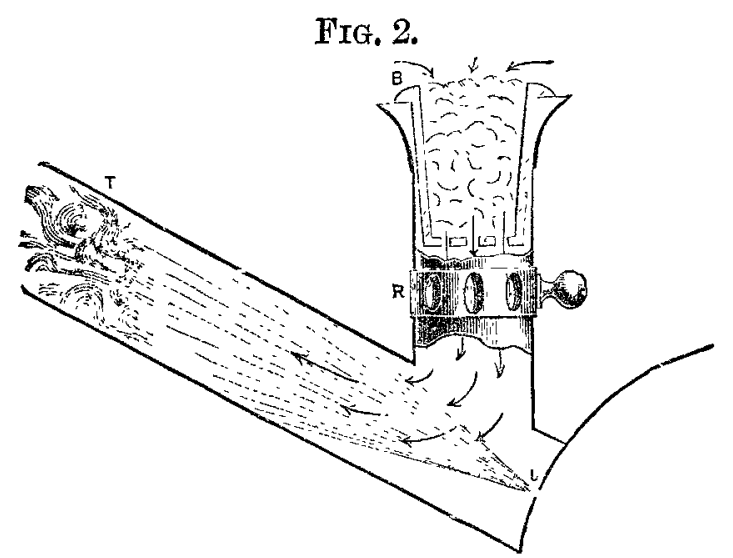

.

represented. A current of air is induced to pass along the tube (T) in the direction of the arrows, and when any substance is placed in the bowl (B) and is ignited, the draught occasioned by the steam jet induces combustion to continue, the rate depending on the force of the jet of steam, by which the velocity or force of the air draught is caused and regulated, and by the quantity of air permitted to pass through the substance.

On some future occasion I may deal with the question of the advantage and disadvantages of treating maladies by the administration of remedies in the form of "fumes." At present we know something of the therapeutic value of opium, stramonium, and a fow other agents in certain forms of pulmonary disorder. My object at present is simply to describe the best mode of obtaining fumes so that one can administer them with ease and accuracy. It appears to me that a wide field of inquiry is opened up to us by the possibility of administering many substances in the form of vapour or fumes, and that this form may in many cases. be preferable to that of the solid or liquid.

In further explanation of the diagram, the use of the ring ( $\mathrm{R}$ ) may be pointed out. Its object is to control the draught by admitting more or less air below the chambar or cup (B), and thus diminish or increase the quantity passing through the ignited substance.

\section{NOTES OF A CASE OF INTRA-CRANIAL TUMOUR.}

By H. MALLiNS, A.B., M.B., M.CH. Univ. Dub.

R. B- - aged thirty-five years, a carpenter by trade, came under observation in the year 1883. His father died, at the age of thirty-five, "of some kind of fits." His mother's age at death, the cause of which was bronchitis, was fiftythree. He was an only child. His wife says that three or four years previously he was a man of the most active habits. imaginable, but that since then a marked change had slowly and gradually come over him : he had lost all his energy and had become quite lazy, and if he sat down on a chair for a minute or so was sure to fall fast asleep. During the year 1883 he came to me on several occasions, complaining of supra-orbital neuralgia of the right side, of no marked severity, the occurrence of which he attributed to the effects of a fall which he had some six years ago, when his forehead was severely bruised. On one of the occasions referred to. he was asked if the pain ever affected the sight of the right eye in any way. He at once replied that it had been bad for a long time. On examination, it was found that vision on that side was nil. The eyeball was slightly more prominent than the left one. The pupils were equal in size, and normal in action. There was no ptosis or strabismus. With the opthalmoscope white atrophy of the right disc was observed, and a similar condition was noticed to be commencing in the left. With the exception of these neuralgic attacks, which commenced three years ago, and the defective vision of the right eye, the patient. seemed perfectly sound. All the functions were normally performed, and there was no indication of loss of power or sensation. The reflexes were normal. The neuralgia yielded to ordinary remedies, and he returned to work. Nothing more was seen of him until Jan. 5th, 1884, when a decided change was noticeable. He seemed dazed, stupid, and remarkably drowsy. This drowsiness was by no means a new feature in his case, though it had much increased of late, and had earned for him amongst his fellow workmon a reputation for laziness which the sequel will prove to have been undeserved, Pulse very slow, 40 . He had had numerous attacks of vomiting and severe occipital pain during a few weeks previously to being seen. The marked degree of drowsiness may be gathered from the fact that it was quite difficult to keep him awake while his eyes were being examined with the ophthalmoscope. The diagnosis of a brain tumour probably situated in the cerebellum was at this time made. On Jan. 23rd, 1884, having so far recovered from. the attacks of pain and vomiting as to be able to travel, he went to the Eye Infirmary at Norwich, where Mr. Robinson kindly examined his eyes with the following result:-Right eye: Vision nil; optic disc atrophied; vessels small. Left: Vision $=\frac{1}{5}$ Jaeger; optic disc displays commencing atrophy: retina congested. During February he suffered a good deal from cough and dyspnœa, apparently of a catarrhal nature. The drowsiness continued, though it was not quite so extreme. A complaint of a curious feeling of weakness-a feeling as if the hip bones would come through the skin"was at this time made. His wife thought he had become rather deaf of late. The following are the notes taken at the time:-

March 4th.-Decided convergent strabismusnoticed to-day. 